\title{
Channel Identifiability Of OFDM-CDMA System Based On Subspace Method
}

\author{
Yonghong Zeng, Member, IEEE, and Tung Sang $\mathrm{Ng}$, Fellow, IEEE \\ Department of Electrical and Electronic Engineering \\ The University of Hong Kong, Hong Kong
}

\begin{abstract}
Subspace method is well-known in CDMA channel estimation. But a basic problem, channel identifiability by subspace method, is still not well-solved. In this paper, two subspace based blind methods for estimating the channel responses of a OFDM-CDMA system in downlink and uplink are discussed respectively. Under some reasonable assumptions, it is mathematically proved that subspace method for downlink can estimate the channel subject to a scalar ambiguity, and the method for uplink can give the channel responses subject to a diagonal matrix amhiguity. The methods do not need precise channel order information (only an upper bound for the orders is required). Simulations show that the methods are effective and robust.

Index Terms- Subspace, OFDM, CDMA, Channel identification
\end{abstract}

\section{INTRODUCTION}

CDMA has been chosen as a main technique for the third generation (3G) wireless communication. On the other hand, orthogonal frequency division multiplexing (OFDM), which can effectively mitigate the effects of multipath propagation and hence increase data rate [1], [2], also emerges as a candidate for wireless communication system beyond 3G. Combining these two techniques therefore have the ability to achieve better performance. So, multi-carrier CDMA, the combination of OFDM and CDMA, has been proposed and studied extensively in recent years [3], [4], [5], [6]. There are various methods for combining the OFDM and CDMA. The so-called MCCDMA is one of them [3], [4]. In the transmitting end of MC.CDMA, each symbol is spread into a block (veotor) of length $N$, and the block is transformed by the inverse discrete Fourier transform (IDFT). The transformed block is then cyclic prefixed or zero-padded to a block of length $M$. In the receiving end, each length- $M$ block is chopped back to length $N$ by discarding some of its first elements or overlapping add. Then the DFT is performed. In the received signal, there are inter-chip interference (ICI) and multi-access interference (MAI). To combat these interferences and recover the transmitted signal, accurate channel information is very helpful and sometimes mandatory.

Subspace methods for CDMA and OFDM-CDMA channel estimation have been studied in recent years $[7],[2],[4],[8],[9],[5],[6],[10]$. Although the methods are tested by some simulations, a basic problem, channel identifiability by subspace method, is still not wellsolved now. The basic idea of a subspace method is that the signal subspace is determined by the eigenvectors of the auto-correlation matrix of received signal. Here the signal subspace is the range space of a structured channel matrix which includes all information of the channel. However, generally speaking, the structured channel matrix is not uniquely determined by its range space. This means that the subspace method may not be able to do cide the channel. Only in some very special cases, the channel can be uniquely (up to some ambiguity) identified by the signal subspace. It is usually difficult to prove the channel identifiability for a subspace method. In this paper, we consider the subspace channel estimation for OFDM-CDMA systems, where zero-padding [11], [2], [12] other than cyclic prefix is used in OFDM. The reason of using zero-padding OFDM (ZP-OFDM) is that it not only has all the advantages of cyclic prefixed OFDM (CP-OFDM) but also avoids inter-block interference (IBI) (in CP-OFDM, there is IBI in the first $L$ elo ments of each received block, where $L$ is the length of the CP, but these $L$ elements is discarded). Without IBI, the channel estimation is simplified [2], [11], [13]. Subspace methods for downlink and uplink are discussed respectively. Under some reasonable assumptions, it is mathematically proved that subspace method for downlink can estimate the channel subject to a scalar ambiguity, and the method for uplink can give the channel responses subject to a diagonal matrix ambiguity. The ambiguity is inherent for any second order statistics (SOS) based method including the subspace method. The methods do not need precise channel onder information (only an upper bound for the orders is required). Simulations show that the methods are effective and robust.

Some notations are used in the following. Superscripts $T, \dagger$ and $*$ stand for transpose, transconjugate, and conjugate, respectively. The symbol diag is used to define a diagonal or block diagonal matrix. 


\section{ZERO-PADDING OFDM-CDMA SYSTEM}

Assume that there are $K$ users in the system and each user is assigned a spreading code $\xi^{(k)}, k=1,2, \cdots, K$. The symbols to be transmitted by user $k$ at time $i$ is $b_{i}^{(k)}, i=0,1, \cdots$. The symbol $b_{i}^{(k)}$ is spread into $N$ $(N \geq K)$ chips which forms a block $\mathbf{s}_{i}^{(k)}=b_{i}^{(k)} \xi^{(k)}$. Now the recently proposed ZP-OFDM [2], [11] is used to modulate each block. In ZP-OFDM, each block is transformed by the inverse discrete Fourier transform (IDFT). After the IDFT, the block is turned to

$$
\mathbf{u}_{i}^{(k)}=\left(u_{i}^{(k)}(0), u_{i}^{(k)}(1), \cdots, u_{i}^{(k)}(N-1)\right)^{T}=b_{i}^{(k)} \mathbf{c}^{(k)}
$$

where

$$
\mathbf{c}^{(k)}=\left(c^{(k)}(0), \cdots, c^{(k)}(N-1)\right)^{T}=\operatorname{IDFT}\left(\xi^{(k)}\right) .
$$

Then $L$ zeros is added to the tail of each block, where $L$ is an upper bound for orders of all channel responses. To assure frequency domain equalization by FFT, it is assumed that $N \geq L$. Each zero-padded block is with length $M=N+L$ and transmitted via the wireless channels.

a. Downlink (base station to mobile). A mobile unit receives a signal (before implementing the DFT) in baseband as

$$
\begin{gathered}
x_{i}(n)=\sum_{k=1}^{K} \sum_{l=0}^{L} h(l) u_{i}^{(k)}(n-l)+\eta_{i}(n), \\
n=\mathbf{0}, \mathbf{1}, \cdots, M-1,
\end{gathered}
$$

where $h(l)$ is the channel response (including the transmitting and receiving filters), $L$ is an overestimated channel order, $\eta_{i}(n)$ is the channel noise, and $u_{i}^{(k)}(n)=$ 0 , if $n<0$ or $N \leq n<M$. Let

$$
\begin{aligned}
\mathbf{b}_{i} & =\left(b_{i}^{(1)}, b_{i}^{(2)}, \cdots, b_{i}^{(K)}\right)^{T}, \\
\mathbf{C} & =\left(\mathbf{c}^{(\mathbf{1})}, \mathbf{c}^{(\mathbf{2})}, \cdots, \mathbf{c}^{(K)}\right), \\
\mathbf{x}_{i} & =\left(x_{i}(\mathbf{0}), x_{i}(\mathbf{1}), \cdots, x_{i}(M-1)\right)^{T}, \\
\eta_{i} & =\left(\eta_{i}(\mathbf{0}), \eta_{i}(\mathbf{1}), \cdots, \eta_{i}(M-\mathbf{1})\right)^{T} .
\end{aligned}
$$

Then (1) can be expressed into a vector form as

$$
\mathbf{x}_{i}=\mathbf{H} \mathbf{C b}_{i}+\eta_{i},
$$

where $\mathbf{H}$ is a $M \times N$ Toeplitz matrix with first column $(h(0), \cdots, h(L), 0, \cdots, 0)^{T}$ and first row $(h(0), 0, \cdots, 0)$.

b. Uplink (mobile to base station). The difference between downlink and uplink is that in downlink there is only one channel (for the desired receiver), while in uplink there are $K$ channels. For synchronized system, the base station receives a signal as

$$
x_{i}(n)=\sum_{k=1}^{K} \sum_{l=0}^{L} \hat{h}^{(k)}(l) u_{i}^{(k)}(n-l)+\eta_{i}(n),
$$

where $\hat{h}^{(k)}(l)\left(l=0,1, \cdots, L_{k}\right)$ is the channel response from user $k$ to base station (including the transmitting and receiving filters). $L$ is an upper bound (maybe overestimated) for all $L_{k}$. If $L_{k}<L$, the channle is zeropadded. By defining

$$
\begin{aligned}
\hat{\mathbf{h}}(l) & =\left(\hat{h}^{(1)}(l), \hat{h}^{(2)}(l), \cdots, \hat{h}^{(k)}(l)\right), \\
\mathbf{u}_{i}(n) & =\left(u_{i}^{(1)}(n), u_{i}^{(\mathbf{2})}(n), \cdots, u_{i}^{(K)}(n)\right)^{T},
\end{aligned}
$$

we can turn (4) into

$$
x_{i}(n)=\sum_{l=0}^{L} \hat{\mathbf{h}}(l) \mathbf{u}_{i}(n-l)+\eta_{i}(n) .
$$

Noticing that $\mathbf{u}_{i}^{(k)}=b_{i}^{(k)} \mathbf{c}^{(k)},(6)$ can be expressed as

$$
\mathbf{x}_{i}=\hat{\mathbf{H}} \hat{\mathbf{C}} \mathbf{b}_{i}+\eta_{i},
$$

where

$$
\begin{gathered}
\hat{\mathbf{C}}=\left(\mathbf{C}_{0}, \mathbf{C}_{1}, \cdots, \mathbf{C}_{N-1}\right)^{T}, \\
\mathbf{C}_{n}=\operatorname{diag}\left(c^{(1)}(n), c^{(2)}(n), \cdots, c^{(K)}(n)\right),
\end{gathered}
$$

and $\hat{\mathbf{H}}$ is a $M \times K N$ block Toeplitz matrix with first block column $\left(\hat{\mathbf{h}}^{T}(0), \cdots, \hat{\mathbf{h}}^{T}(L), 0, \cdots, 0\right)^{T}$ and first row $(\hat{\mathbf{h}}(0), 0, \cdots, 0)$. Please note that here $\hat{\mathbf{h}}(l)$ is a $\mathbf{1} \times K$ matrix, while in (1) $h(l)$ is a scalar.

Given that the noises can be ignored in (3) or (7), $\mathbf{b}_{i}$ is completely recoverable from $x_{i}$, that is, there exists a zero-forcing equalizer, if and only if matrix $\mathbf{H C}$ or $\hat{\mathbf{H}} \hat{\mathbf{C}}$ is of full column rank. In (3), it is obvious that if $h(0) \neq 0$, HC if of full column rank. The condition for $\hat{\mathbf{H}} \hat{\mathbf{C}}$ to be of full column rank is much more complicated. The full column rank condition is assumed in the following.

\section{SUBSPACE ALGORITHM FOR DOWNLINK}

The following conditions for the statistical properties of transmitted symbols $b_{l}(k)$ and channel noise samples $\eta_{i}(n)$ are assumed.

(A1) Noise samples are white, uncorrelated and have identical distributions.

(A2) Noise samples and transmitted signals are uncorrelated, that is, $\mathbf{E}\left(\eta_{i}(n)\left(b_{l}(k)\right)^{*}\right)=\mathbf{0}$.

Here $\mathbf{E}(y)$ means the mathematical expectation of a random variable $y$.

Based on the assumptions (A1) and (A2), we can verify that $\mathbf{R}_{x}=\mathbf{E}\left(\mathbf{x}_{i} \mathbf{x}_{i}^{\dagger}\right)=\mathbf{H C} \mathbf{R}_{b} \mathbf{C}^{\dagger} \mathbf{H}^{\dagger}+\sigma_{\eta}^{2} \mathbf{I}_{M}$, where $\mathbf{R}_{b}=\mathbf{E}\left(\mathbf{b}_{i} \mathbf{b}_{i}^{\dagger}\right)$ is a positive definite matrix. The smallest eigenvalue of matrix $\mathbf{R}_{x}$ is $\sigma_{\eta}^{2}$. Since the rank of $\mathbf{H C R}_{\mathrm{b}} \mathbf{C}^{\dagger} \mathbf{H}^{\dagger}$ is $K$, there are $q=M-K$ co-orthogonal eigenvectors corresponding to the smallest eigenvalue. These eigenvectors are denoted by $\beta_{i}(i=0,1, \cdots, q-1)$. Based on simple mathematical derivation which is used 
in standard subspace method, we know that $\beta_{i}^{\dagger} \mathbf{H C}=$ $0, i=0,1, \cdots, q-1$. Therefore, the vector space spanned by the column vectors of matrix $\mathbf{H C}$ is uniquely determined. For simplicity, we use span(HC) to denote the vector space spanned by the column vectors of $\mathbf{H C}$, that is, all possible linear combinations of the column vectors. In general, knowing span(HC) cannot determine matrix HC. However, for some matrix with special structure, it is possible to determine HC by span(HC) up to certain ambiguity. In the following, we give the following assumption for the spreading codes:

(A3) $\operatorname{GCD}\left(C^{(1)}(z), C^{(2)}(z), \cdots, C^{(K)}(z)\right)=1$, where

$$
C^{(k)}(z)=\sum_{n=0}^{N-1} c^{(k)}(n) z^{n}, k=1,2, \cdots, K,
$$

where GCD means Greatest Common Divisor. In the following, we call $C^{(k)}(z)$ the spreading polynomial of user $k$ because it depends only on the spreading code of the user. Under the assumption, the following theorem states that $\mathbf{H C}$ is uniquely determined by $\operatorname{span}(\mathbf{H C})$ subject to a scalar ambiguity.

Theorem 1: Let $\mathbf{H}$ be the matrix in (3) and $\overline{\mathbf{H}}$ be a matrix which has the same form as $\mathbf{H}$ with $h(l)$ being replaced by $\bar{h}(l)$. Assume that $h(0) \neq 0$ and $\bar{h}(0) \neq 0$. If $\operatorname{span}(\mathbf{H C})=\operatorname{span}(\overline{\mathbf{H}} \mathbf{C})$, then there exists a scalar $\lambda$ such that $\mathbf{H C}=\lambda \overline{\mathbf{H}} \mathbf{C}$.

Proof. It is easy to verify that $\operatorname{span}(\mathbf{H C})=\operatorname{span}(\overline{\mathbf{H} C})$ if and only if there exists an $K \times K$ invertible matrix $\mathbf{A}=\left(a_{i j}\right)$ such that $\mathbf{H C}=\overline{\mathbf{H}} \mathbf{C A}$. We section the matrix $\mathbf{C}$ into $\mathbf{C}=\left(\mathbf{d}_{0}^{T}, \mathbf{d}_{1}^{T}, \cdots, d_{N-1}^{T}\right)^{T}$, where $d_{n}$ is a $1 \times$ $K$ matrix. From the definition of matrix $C$, we know that $\mathbf{d}_{n}=\left(c^{(1)}(n), \cdots, c^{(K)}(n)\right)$. Now, we turn equation $\mathbf{H C}=\overline{\mathbf{H} C A}$ into an equivalent, form as

$$
\sum_{l=0}^{L} h(l) \mathbf{d}_{n-l}=\sum_{l=0}^{L} \bar{h}(l) \mathbf{d}_{n-l} \mathbf{A} .
$$

By defining

$$
H(z)=\sum_{l=0}^{L} h(l) z^{l}, \bar{H}(z)=\sum_{l=0}^{L} \bar{h}(l) z^{l},
$$

and

$$
\mathbf{D}(z)=\sum_{n=0}^{N-1} \mathbf{d}_{n} z^{n}
$$

we get an equivalent form for (9) as

$$
H(z) \mathbf{D}(z)=\vec{H}(z) \mathbf{D}(z) \mathbf{A} .
$$

From the definition of $\mathbf{D}(z)$, we have

$$
\begin{aligned}
\mathbf{D}(z) & =\sum_{n=0}^{N-1}\left(c^{(1)}(n), c^{(2)}(n), \cdots, c^{(K)}(n)\right) z^{n} \\
& =\left(C^{(1)}(z), C^{(2)}(z), \cdots, C^{(K)}(z)\right) .
\end{aligned}
$$

Therefore, we get $H(z) C^{(k)}(z)=\bar{H}(z) \mathbf{D}(z) \mathbf{A}_{k}$, where $A_{k}$ is the $k$ th column of matrix A. So, $\bar{H}(z) \mid H(z) C^{(k)}(z)(k=1,2, \cdots, K)$, which is equivalent to

$\bar{H}(z) \mid H(z) \operatorname{GCD}\left(C^{(1)}(z), C^{(2)}(z), \cdots, C^{(K)}(z)\right)=H(z)$,

where | means dividing in number theory. Since $\mathbf{A}$ is invertible, we can also prove that $H(z) \mid \bar{H}(z)$. Hence, there exists a scalar $\lambda \neq 0$ such that $H(z)=\lambda \bar{H}(z)$. So, we have $\lambda \mathbf{D}(z)=\mathbf{D}(z) \mathbf{A}$, that is,

$$
\begin{gathered}
\lambda C^{(k)}(z)=a_{1 k} C^{(1)}(z)+a_{2 k} C^{(2)}(z)+\cdots+a_{K k} C^{(K)}(z), \\
k=1,2, \cdots, K,
\end{gathered}
$$

which can be written into vector form as

$$
\begin{gathered}
\lambda \mathbf{c}^{(k)}=a_{1 k} \mathbf{c}^{(1)}+a_{2 k} \mathbf{c}^{(2)}+\cdots+a_{K k} \mathbf{c}^{(K)}, \\
k=1,2, \cdots, K .
\end{gathered}
$$

Since $\mathrm{c}^{(k)}(k=1,2, \cdots, K)$ are linear independent, we know that $a_{k k}=\lambda, a_{j k}=\mathbf{0}(j \neq k)$, that is, $\mathbf{A}=\lambda \mathbf{I}_{K}$, or $\mathbf{H C}=\lambda \overline{\mathbf{H}} \mathbf{C}$.

The physical meaning of assumption (A3) is that there is not a common zero for the spreading polynomials of the $K$ users, which is easy to satisfy in practice. Assumption (A3) has nothing to do with the channel, that is, by properly choosing the spreading codes, the subspace method is valid in any channel conditions. Even if the assumption (A3) is not satisfied, theorem 1 still holds obviously if $H(z)(\bar{H}(z))$ is relatively prime with $\operatorname{GCD}\left(C^{(1)}(z), C^{(2)}(z), \cdots, C^{(K)}(z)\right)$, that is, the channel does not have a common zero with at least one spreading polynomial, which is almost surely (with probability one) guaranteed for random channels.

\section{SUBSPACE ALGORITHM FOR UPLINK}

Like the downlink case, a subspace method can only determine $\operatorname{span}(\hat{\mathbf{H}} \hat{\mathbf{C}})$. A tough task is to study if $\hat{\mathbf{H}} \hat{\mathbf{C}}$ can be uniquely determined (up to some ambiguity) from $\operatorname{span}(\hat{\mathbf{H}} \mathbf{C})$. In general, if we have another possible channel matrix $\tilde{\mathbf{H}}$, which has the same form as $\hat{\mathbf{H}}$ with $\hat{\mathbf{h}}(l)$ replaced by $\tilde{\mathbf{h}}(l)$, such that $\operatorname{span}(\hat{\mathbf{H}} \hat{\mathbf{C}})=\operatorname{span}(\hat{\mathbf{H}} \hat{\mathbf{C}})$, we can only say that there exists an invertible matrix $\mathbf{A}$ such that $\hat{\mathbf{H}} \hat{\mathbf{C}}=\overline{\mathbf{H}} \hat{\mathbf{C}} \mathbf{A}$. From the definitions of the matrices, we can turn this condition into a equivalent form as

$$
\hat{\mathbf{H}}(z) \mathbf{C}(z)=\overline{\mathbf{H}}(z) \mathbf{C}(z) \mathbf{A}
$$

where

$$
\hat{\mathbf{H}}(z)=\sum_{l=0}^{L} \hat{\mathbf{h}}(l) z^{l}, \tilde{\mathbf{H}}(z)=\sum_{l=0}^{L} \tilde{\mathbf{h}}(l) z^{l}
$$


and $\mathbf{C}(z)=\operatorname{diag}\left(C^{(1)}(z), C^{(2)}(z), \cdots, C^{(K)}(z)\right)$. Please note that here $\hat{\mathbf{H}}(z)$ and $\tilde{\mathbf{H}}(z)$ are $1 \times K$ polynomial matrix. So, there is a big difference between (10) and (15). By defining $\mathbf{E}_{k}$ to be a $M \times(L+1)$ Toeplitz matrix with first column $\left(c^{(k)}(0), \cdots, c^{(k)}(N-1)\right)^{T}$ and first row $\left(c^{(k)}(0), 0, \cdots, 0\right)$, and

$$
\begin{aligned}
& \hat{\mathbf{h}}^{(k)}=\left(\hat{h}^{(k)}(0), \hat{h}^{(k)}(\mathbf{1}), \cdots, \hat{h}^{(k)}(L)\right)^{T}, \\
& \tilde{\mathbf{h}}^{(k)}=\left(\tilde{h}^{(k)}(0), \tilde{h}^{(k)}(1), \cdots, \tilde{h}^{(k)}(L)\right)^{T},
\end{aligned}
$$

we turn (15) into

$$
\begin{gathered}
\mathbf{E}_{k} \hat{\mathbf{h}}^{(k)}=a_{1 k} \mathbf{E}_{1} \tilde{\mathbf{h}}^{(1)}+a_{2 k} \mathbf{E}_{2} \tilde{\mathbf{h}}^{(2)}+\cdots+a_{K k} \mathbf{E}_{K} \tilde{\mathbf{h}}^{(K)}, \\
k=\mathbf{1}, 2, \cdots, K .
\end{gathered}
$$

Now we set another assumption for the spreading codes:

(A4) For any length- $(L+1)$ non-zero vectors $\mathrm{f}^{(k)}$ $(k=1,2, \cdots, K), \mathbf{E}_{k} \mathbf{f}^{(k)}(k=1,2, \cdots, K)$ are linear independent.

Under this assumption, it is easy to show that the coefficients $a_{j k}$ in (16) must satisfy $a_{j k}=0$ for $j \neq k$, that is, $\mathbf{A}$ must be a diagonal matrix. If $\mathbf{A}$ is diagonal, we have $\mathbf{C}(z) \mathbf{A}=\mathbf{A C}(z)$ and therefore $\hat{\mathbf{H}}(z)=\tilde{\mathbf{H}}(z) \mathbf{A}$. So, the subspace method can determine the channel $\hat{\mathbf{H}}(z)$ subject to a diagonal matrix ambiguity.

Theorem 2: Under assumption (A4), the subspace method can determine the channel matrix subject to a diagonal matrix ambiguity, that is, $\hat{\mathbf{h}}(l)=\overline{\mathbf{h}}(l) \mathbf{A}$, where $\tilde{\mathbf{h}}(l)$ is the estimated channel, $\mathbf{A}$ is a diagonal matrix and $l=0,1, \cdots, L$.

The assumption is only imposed on the spreading codes and has nothing to do with the channels. However, the physical meaning of it remains to be investigated further.

\section{Simulations}

In the following, $\mathbf{R}_{x}$ is computed by $\mathbf{R}_{x}=\mathbf{E}\left(\mathbf{x}_{i} \mathbf{x}_{i}^{\dagger}\right) \approx$ $\frac{1}{L_{s}} \sum_{i=0}^{L_{s}-1} \mathbf{x}_{i} x_{i}^{\dagger}$, where $L_{s}$ is the number of block samples used. The columns of Hadamard matrix are used as spreading codes. Signal-noise-ratio (SNR) means the ra tio of the received signal power with the noise power as

$$
\mathrm{SNR}=\frac{\mathbf{E}\left(\left|x_{i}(n)-\eta_{i}(n)\right|^{\mathbf{2}}\right)}{\mathbf{E}\left(\left|\eta_{i}(n)\right|^{2}\right)}
$$

The subspace methods leave a scalar or diagonal matrix ambiguity respectively for downlink and uplink, which cannot be resolved by SOS based blind method. For the purpose of verification, it is obtained as follows. For downlink, let

$$
\lambda=\sum_{l=0}^{L-1} \tilde{h}^{*}(l) h(l) / \sum_{l=0}^{L-1} \tilde{h}^{*}(l) \tilde{h}(l),
$$

and for uplink, let

$$
\left.a_{k}=\sum_{l=0}^{L-1}\left[\tilde{h}^{(k)}\right)(l)\right]^{*} \hat{h}^{(k)}(l) / \sum_{l=0}^{L-1}\left[\tilde{h}^{(k)}(l)\right]^{*} \tilde{h}^{(k)}(l)
$$

and $\mathbf{A}=\operatorname{diag}\left(a_{1}, \cdots, a_{K}\right)$. The estimated channel is then computed by $\bar{h}(l)=\lambda \tilde{h}(l)$ (for downlink) and $\overline{\mathbf{h}}(l)=\tilde{\mathbf{h}}(l) \mathbf{A}$ (for uplink), $l=\mathbf{0}, \mathbf{1}, \cdots, L-1$. Here $\tilde{h}(l)$ and $\tilde{h}^{(k)}(l)$ are the obtained channel responses from the subspace method for downlink and uplink, respectively. The true channel coefficients are used here only for verification and never be used elsewhere. The normalized mean square error (NMSE) between the estimated and true channel responses is defined as

$$
\text { NMSE }=\frac{\sum_{l=1}^{L}|\bar{h}(l)-h(l)|^{2}}{\sum_{l=1}^{L}|h(l)|^{2}}(\text { for downlink })
$$

or

$$
\text { NMSE }=\frac{\sum_{l=1}^{L}\|\overline{\mathbf{h}}(l)-\hat{\mathbf{h}}(l)\|^{2}}{\sum_{l=1}^{L}\|\hat{\mathbf{h}}(l)\|^{2}}(\text { for uplink }) .
$$

Simulations show that the algorithms are effective. Two examples are shown below. The results are averaged over 100 Monte Carlo test.

Example 1: In downlink, $K=N=32$ and $M=48$. The channel responses $h(l)$ are generated randomly, where the true channel order is $\mathbf{1 2}$ and it is overestimated to $L=16$. The transmitted baseband signals are 4-QAM. The number of received block for computing $\mathbf{R}_{z}$ is 70 . Figure 1 shows the NMSE of the estimated channel versus SNR.

Example 2: In uplink, $K=N=8$ and $M=13$. The transmitted baseband signals are 64-QAM. The channel responses $\hat{\mathbf{h}}(l)$ are generated randomly, where the true channel orders (different for different users) are not greater than 4 and they are overestimated to $L=5$. 50 received blocks are used for computing $\mathbf{R}_{x}$. The NMSE of the estimated channels versus SNR is shown in Figure 2.

\section{Conclusions}

Subspace methods have been proposed for estimating the channel responses of a zero-padding OFDM-CDMA system. Under some reasonable assumptions, it is mathematically proved that subspace method for downlink can estimate the channel subject to a scalar ambiguity, and the method for uplink can give the channel responses subject to a diagonal matrix ambiguity. Owing to zero-padding, the subspace methods do not need precise channel order information (only an upper bound for the orders is required). Simulations show that the methods are effective and robust. 


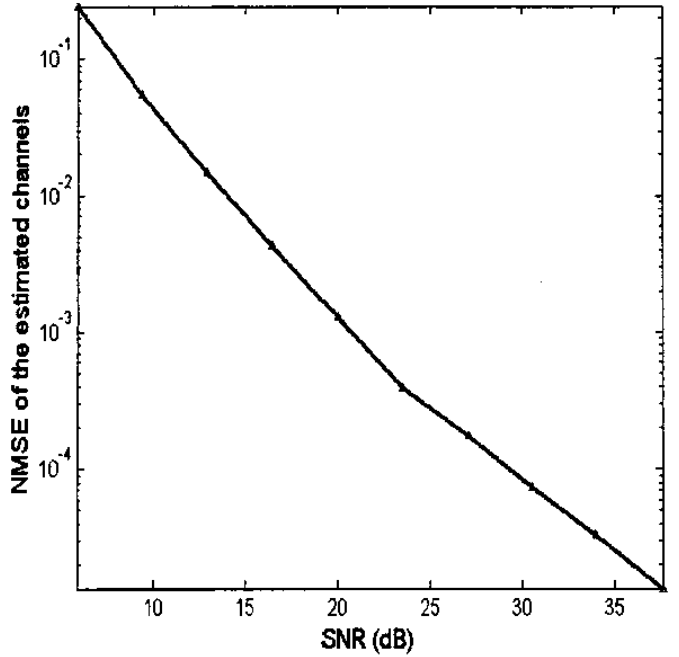

Fig. 1. NMSE versus SNR (downlink)

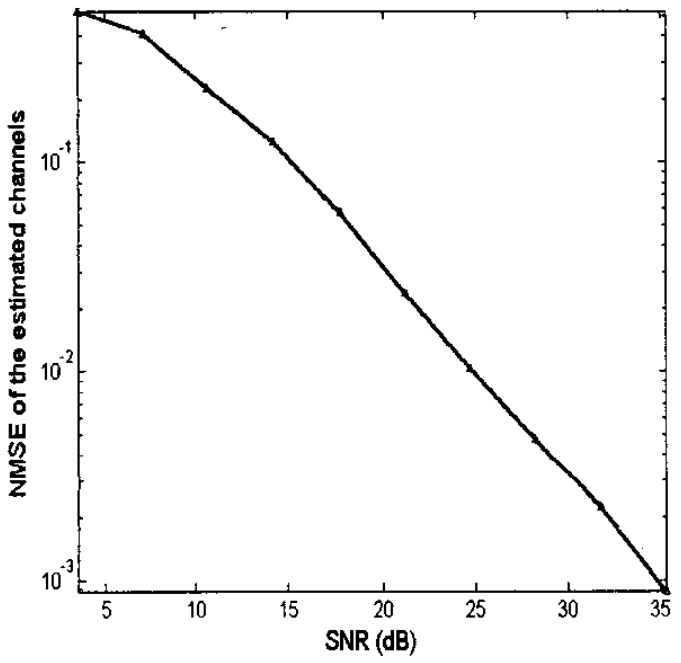

Fig. 2. NMSE versus SNR (uplink)

\section{REFERENCES}

[1] J. Chuang and N. Sollenberger, "Beyond 3G: wideband wireless data acoess based on OFDM and dynamic packet assignment," IEEE Communication Magavine, vol. 32, no. 1, pp. $78-87,2000$.

[2] G. B. Giammakis, Y. Hua, P. Stoica, and L. Tong, Signal Processing Advances in Wireless $\&$ Mobile Communications, vol. 1: Trends in Channel Estimation and Equalisation. PTR: Prentice Hall, 2001.

[3] S. Hara and R. Prasad, "Overview of multicarrier CDMA," IEEE Communication Magasine, pp. 126-133, Dec. 1997.

[4] H. Liu, Signal processing applications in CDMA communications. Boston, London: Artech House Publishers, 2000.

[5] A.-J. V. D. Veen, "Blind subspace-based uplink receiver algorithm for wideband CDMA," in IEEE Vehicular Technology
Conference (VTC'99), vol. 1, pp. 176-180, 1999.

[6] J. Wu, Y. Wang, and K. K. M. Cheng, "Blind chamel estimation based on subspace for multicarrier CDMA," in IEEE Vehicular Techrology Conference (VTC'01), vol. 1, pp. 23742378, 2001.

[7] S. Bensley and B. Aazhang, "Subspace-based charmel estimation for code division multiple access communication systems," IEEE Trans. Communications, vol. 44, no. 8, pp. 1009-1020, 1996.

[8] H. Liu and G. Xu, "A subspace method for signature waveform estimation in synchronous CDMA systems," IEEE Thans. Communications, vol. 44, no. 10, pp. 1346-1354, 1996.

[9] P. Loubaton and E. Moulines, "On blind multiuser forward link chanmel estimation by the subspace method: identifiability results," IEEE Trans. Signal Processing, vol. 48 , no. 8 , pp. 2366-2376, 2000.

[10] Z. Xu, "Asymptotic performance of subspace methods for synchronous multirate CDMA systems," IEEE Trans. Signal Processing, vol. 50, no. 8, pp. 2015-2026, 2002.

[11] A. Scaglione, G. B. Giamnakis, and S. Barbarassa, "Redundant filterbank precoders and equalizers-part ii: blind chanmel estimation, synchronization and direct equalization," IEEE Trans. Signal Processing, vol. 47, no. 7, pp. 2007-2022, 1999.

[12] B. Muquet, Z. Wang, G. B. Giannakis, M. D. Courville, and P. Duhamel, "Cyclic prefixing or zero padding for wireless multicarrier transmissions," IEEE Trans. Communications, vol. 50, no. 12, pp. 2136-2148, 2002.

[13] Y. H. Zeng and T. S. Ng, "A semi-blind chamnel estimation method for multi-user multi-anterma OFDM systemus," IEEE Trans. Signal Processing, vol. 51, 2003. 